\title{
Real-life experience: sensitivity and specificity of nasal and saliva samples for COVID-19 diagnosis
}

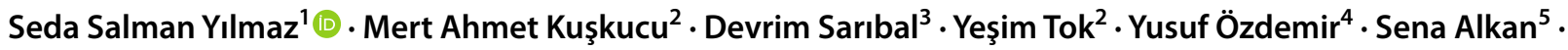 \\ Hatice Yaşar Arsu ${ }^{5} \cdot$ Metin Yalçın ${ }^{5}$. Okan Nohut ${ }^{6}$. Illker İnanç Balkan ${ }^{5} \cdot$ Gökhan Aygün $^{5} \cdot$ Kenan Midilli $^{2}$
}

Received: 15 September 2021 / Accepted: 22 October 2021 / Published online: 3 November 2021

(c) The Author(s), under exclusive licence to Royal Academy of Medicine in Ireland 2021

\begin{abstract}
Background COVID-19 (coronavirus disease 2019) outbreak has spread rapidly around the world, continues to show its effect, and it is not clear how long it will continue. For the diagnosis of COVID-19, it is important to ensure the comfort of the patients and to protect the healthcare workers (HCWs) by reducing the use of protective equipment.

Aims To evaluate or assess whether the samples taken by the patient for COVID-19 testing during this pandemic period can be used in real-life experience.

Methods Three different samples (nasopharyngeal taken by the healthcare worker, nasopharyngeal, and saliva taken by the patient) from 132 patients were evaluated for the diagnosis of COVID-19. The sensitivity and specificity of the samples in the diagnosis of COVID-19 were compared with real-life experience.

Results Paired analyzes were performed by comparing each sample taken by the healthcare worker with the sample taken by the patient. The sensitivity of the three samples (nasopharyngeal taken by the healthcare worker, nasopharyngeal, and saliva taken by the patient) in the diagnosis of the COVID-19 was $(100 \%, 98.7 \%$, and $96.1 \%$, respectively) accepted to be accurate. Conclusions The sample taken by the paramedic was compatible compared to the real-life experience for the samples taken by the patient in the COVID-19 pandemic period. During the pandemic that is unknown when it will end, this study demonstrated that taking the sample of the patient alone for the severe acute respiratory syndrome coronavirus 2 (SARS-CoV-2) test is a beneficial approach to the protection of the healthcare worker, reducing the need for protective equipment, increasing the patient's comfort and rapid sampling.
\end{abstract}

Keywords COVID-19 $\cdot$ Healthcare worker $\cdot$ Real-life experience $\cdot$ Sensitivity $\cdot$ Specificity

Seda Salman Yilmaz

salmanseda@gmail.com

1 Cerrahpasa Faculty of Medicine, Department of COVID-19 Diagnosis Laboratory, Istanbul University-Cerrahpaşa, Istanbul, Turkey

2 Cerrahpasa Faculty of Medicine, Department of Medical Microbiology, Istanbul University-Cerrahpaşa, Istanbul, Turkey

3 Cerrahpasa Faculty of Medicine, Department of Biophysics, Istanbul University-Cerrahpaşa, Istanbul, Turkey

4 Specialist of Infectious Diseases and Clinical Microbiology, Ministry of Health Sadi Konuk Education Research Hospital, Bakırköy, Istanbul, Turkey

5 Cerrahpasa Faculty of Medicine, Department of Infectious Diseases and Clinical Microbiology, Istanbul University-Cerrahpaşa, Istanbul, Turkey

6 Cerrahpasa Faculty of Medicine, Department of Medical Biochemistry, Istanbul University-Cerrahpaşa, Istanbul, Turkey

\section{Introduction}

Since December 2019, the causative of the coronavirus disease 2019 (COVID-19) is spreading rapidly around the world. Globally, there have been $229,437,517$ cases, 4,879,235 deaths reported to The World Health Organization (WHO) [1]. COVID-19 pandemic is an ongoing devastating threat to human lives and livelihoods worldwide. Healthcare workers (HCWs) are an important part of the front lines in the fight against the pandemic. Many HCWs have been infected with severe acute respiratory syndrome coronavirus 2 (SARS-CoV-2) and died worldwide during the pandemic. According to $\mathrm{WHO}$, at least $50 \%$ of patients who died of COVID-19 were residents in hospitals or nursing homes, which highlights the need to control the spread of infection in a healthcare setting [2].

Remarkably, as pandemic accelerates, access to personal protective equipment (PPE) is a major challenge for 
healthcare workers. HCWs are also concerned about carrying the infection from one patient to another, as well as having personal safety concerns, and passing the infection to their families. The most likely risk of carrying family members, elderly parents, or young children to older parents [3, 4]. HCWs have always been easy targets for the transmission of infectious diseases like COVID-19. Protecting HCWs is of consequential significance in ensuring continued medical treatment for the whole population in reducing further spread [5].

On the other clinical conditions, it is faster to obtain a tongue, nasal, or mid-turbinate sample than a nasopharyngeal sample and it is less potential for the patient to sneeze, cough, or gag. Also, recent data support the validity of nonnasopharyngeal samples for the detection of SARS-CoV-2. Collection by the patient reduces the high exposure of the healthcare worker to the virus and preserves limited PPE. During the swab sampling process, droplets and aerosol are produced by sneezing, coughing, gagging reflexes and talking of patients, and close contact between healthcare workers. Therefore, COVID-19 patients may directly cause infections in HCWs $[6,7]$.

Recent research has shown the accuracy of nonnasopharyngeal samples for SARS-CoV-2. Sampling by the patient reduces the high exposure of the healthcare worker, reduces personnel equipment use, and increases access to testing. Currently, the diagnosis of COVID-19 mainly depends on the real-time RT-PCR test of the upper respiratory tract sampling in clinical conditions, and it is faster to minimize the risk of exposure to the healthcare workers, and reduce personnel equipment use to testing [8].

In this study, we compared the saliva sample, healthcare worker collected nasal and throat swab samples, and patient-collected nasal swab samples for the diagnosis of COVID-19.

\section{Material and method}

\section{Sample collection}

This study was conducted among 132 individuals. Outpatients with symptoms who were admitted to the COVID-19 Clinic between 01 April and 05 June 2020 were included in our study. All samples were obtained after receiving the written informed consent of participants. Upper respiratory samples were collected from patients who were presented with a history of fever or multiple acute respiratory symptoms, history of contact with a positive individual, or travel history from the endemic area of COVID-19 within 14 days. In this cross-sectional study, three different upper respiratory samples (nasopharyngeal taken by the healthcare worker, nasopharyngeal, and saliva taken by the patient) were collected. In addition to the samples taken by the health worker in our study, visual posters were prepared for the samples taken from the patients themselves and explained with oral instructions. Three different samples were taken in a biosafety cabinet and decontamination was performed after each patient. In the study, samples were collected by a single person for standardization, and nasal samples were collected with a flocked swab. Nasopharyngeal and throat swabs were collected using Puritan Liquid-based Specimen Collection and Transport Systems and saliva samples were collected using sterile sample containers. Clinical and demographic information of the overall patients $(n=132)$, including age, gender, symptoms, comorbidities, and coinfections were provided in Table 1.

\section{Specimen processing}

Upper respiratory samples were labeled with different numbers and collected using sterile tubes containing universal transport medium and sterile sample containers. Immediately, viral RNA was extracted using GENEALL RNA Isolation Kit (Seoul, South Korea) according to the manufacturer's protocol.

Table 1 Demographics and clinical symptoms

\begin{tabular}{|c|c|}
\hline \multicolumn{2}{|l|}{ Overall $(n=132)$} \\
\hline COVID-19, n (\%) & $76(57.6)$ \\
\hline Age (years), mean (SD) & $38.0( \pm 12.5)$ \\
\hline Male, $\mathbf{n}(\%)$ & $72(54.5)$ \\
\hline Close contact, $\mathbf{n}(\%)$ & $81(61.4)$ \\
\hline Smoking, n (\%) & $42(31.8)$ \\
\hline Healthcare personnel, n (\%) & $10(7.6)$ \\
\hline \multicolumn{2}{|l|}{ Comorbidities } \\
\hline Hypertension, n (\%) & $13(9.8)$ \\
\hline Diabetes, n (\%) & $15(11.4)$ \\
\hline Asthma, n (\%) & $8(6.1)$ \\
\hline \multicolumn{2}{|l|}{ Symptoms at presentation } \\
\hline Fever, n (\%) & $43(32.5)$ \\
\hline Cough, n (\%) & $53(40.2)$ \\
\hline Sore throat, n (\%) & $41(31.1)$ \\
\hline Dyspnoea, n (\%) & $15(11.4)$ \\
\hline Myalgia, n (\%) & $65(49.2)$ \\
\hline Headache, n (\%) & $59(44.7)$ \\
\hline Nausea, n (\%) & $18(13.6)$ \\
\hline Vomiting, $\mathbf{n}(\%)$ & $8(6.1)$ \\
\hline Diarrhea, n (\%) & $16(12.1)$ \\
\hline Fatigue, n (\%) & $78(59.1)$ \\
\hline Hiposmia, n (\%) & $27(20.5)$ \\
\hline Loss of taste, $\mathrm{n}(\%)$ & $28(21.2)$ \\
\hline
\end{tabular}

(Abbreviations: $S D$ standard deviation, COVID-19 coronavirus disease 2019) 
From each sample, detection of SARS-CoV-2 was performed by RT-PCR amplification of the SARS-CoV-2 RdRp and $\mathrm{N}$ gene regions. The SARS-CoV-2 reactions were performed using GeneMark Real-Time Kit (Carlsbad, CA, USA) and the detection of the human RNase P gene was included in the kit as a control. RT-PCR reactions were evaluated in a CFX96 Real-Time Detection System (Biorad, Hercules, CA, USA). The reactions were carried out as follows: 1 cycle of $50{ }^{\circ} \mathrm{C}$ for $30 \mathrm{~min}$, then 1 cycle of $95^{\circ} \mathrm{C}$ for $15 \mathrm{~min}$, followed by 40 cycles of $95^{\circ} \mathrm{C}$ for $15 \mathrm{~s}$ and $58{ }^{\circ} \mathrm{C}$ for $1 \mathrm{~min}$.

\section{Statistical analysis}

Quantitative variables were expressed as mean and standard deviation if they contain continuous data. If they contain categorical data, they are expressed as a percentage (\%) and frequency (n). A comparison of qualitative variables was analyzed with the Pearson Chi-square test.

The normal distribution, which was used to question the presence of parametric data in the data containing the measurement, was examined by Kolmogorov-Smirnov and Kurtosis-Skewness Tests. Parametric tests such as Student's t-test were used for normally distributed data. Kruskal-Wallis Test was used for the analysis of continuous and more than two independent non-parametric groups (Bonferroni correction was used when necessary) and Mann-Whitney Test was used for post-hoc analysis.

The results were evaluated with a $95 \%$ confidence interval and the statistical significance level was defined as $\mathrm{p}<0.05$. The analyses were performed using IBM SPSS-21 (Statistical Package for Social Sciences, Chicago, IL, USA).

Four separate analyses were performed using the Bland \& Altman methodology: the first of them comparing nasopharyngeal samples to sampled health-worker samples for $\mathrm{N}$ gene and RdRp gene. The second comparing saliva samples to health-worker samples for the $\mathrm{N}$ gene and RdRp gene (Fig. 1). Samples were analyzed using RT-PCR and Ct values below 38 were accepted as positive results. In the study, Ct values above 35 in the RNase $\mathrm{P}$ gene were considered negative and were completely excluded from the study. In addition, Chi-squared, Woolf's test and Mantel-Haenszel were used to compare samples by period and evaluate their significance.

\section{Results}

In this study, 132 patients' results of nasopharyngeal (patient and health worker) and saliva samples were evaluated. The average age of the patients was $38.0( \pm 12.5)$ and seventytwo $(54.5 \%)$ individuals were men. All the symptoms occurred for a maximum of 2 to 5 days. Common symptoms fatigue (78 participants $59.1 \%$ ), myalgia (65 participants
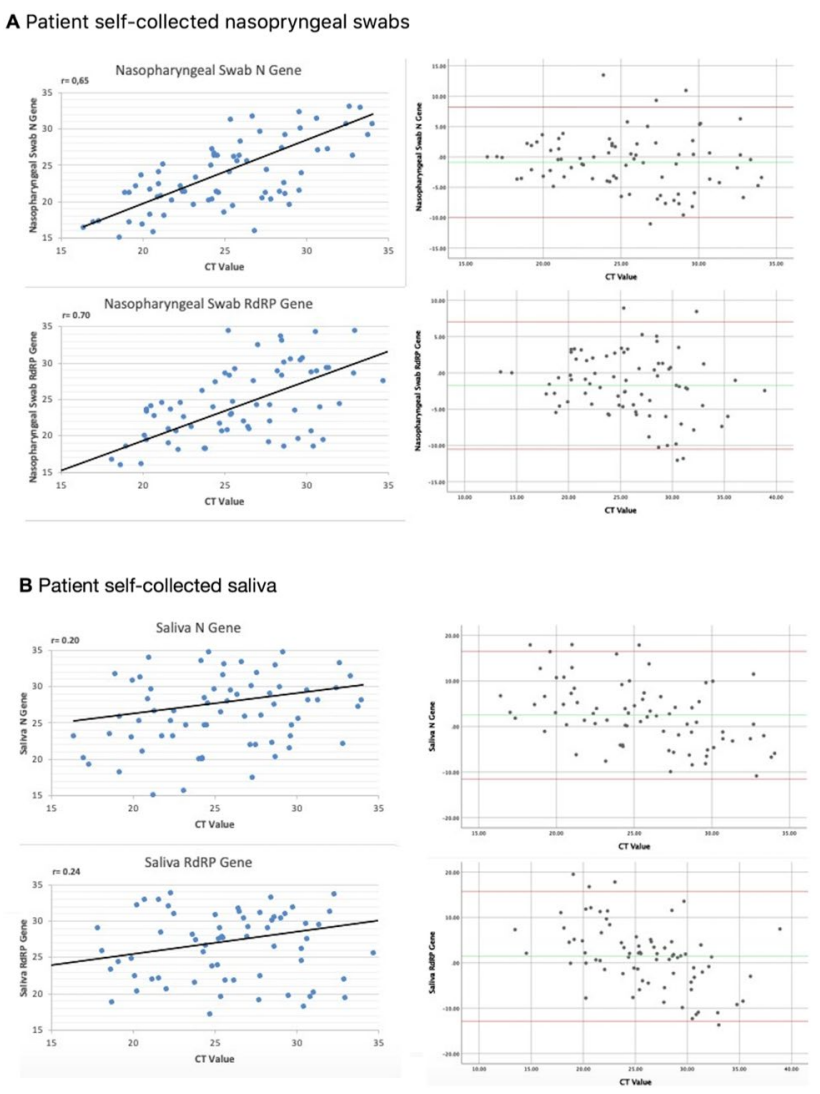

Fig. 1 COVID-19 N gene and RdRp gene cycle threshold $(\mathrm{Ct})$ values from nasopharyngeal swabs and saliva samples by patients to nasopharyngeal swabs and saliva samples by healthcare workers

(49.2\%), headache (59 participants (44.7\%), sore throat (41 participants $(31.1 \%)$, and cough $53(40.2 \%)$ were mostly reported during the first clinical visit. Overall patients' characteristics and clinical informations were summarized in Table 1, and COVID-19 positive patients' characteristics and clinical informations were summarized in Table 2 . We were diagnosed, 76 of 132 patients with COVID-19 by nasopharyngeal swab (taken by the healthcare worker and the patient) or by RT-PCR from saliva samples. RT-PCR results were positive in all samples taken by healthcare worker, nasopharyngeal swab samples (NSS) taken by the patient in 75 patients, and saliva samples in 73 patients. Table 3 summarized the findings of all samples results presented in the current study.

The Pearson Correlation (r-value) results between the $\mathrm{Ct}$ values of the NSS and saliva samples taken by the patient and the NSS Ct values taken by the healthcare worker were $0.65,0.20$, respectively, for the $\mathrm{N}$ gene; the RdRP gene results were $0.70,0.24$ (Fig. 1). Also, Fig. 1 shows the correlation values between the samples taken by the healthcare worker and the samples taken by the patient; $65 \%$ in the $\mathrm{N}$ gene, $70 \%$ in the RdRP gene of nasopharyngeal samples; Saliva was detected as $24 \%$ in the RdRP gene. The mean 
Table 2 Demographic characteristics and clinical symptoms of COVID-19 positive patients

\begin{tabular}{|c|c|}
\hline \multicolumn{2}{|l|}{ COVID-19 $(n=76)$} \\
\hline Age (years), mean (SD) & $38.1( \pm 13.1)$ \\
\hline Male, $\mathbf{n}(\%)$ & $42(55.3)$ \\
\hline Close contact, $\mathbf{n}(\%)$ & $52(68.4)$ \\
\hline Smoking, n (\%) & $17(22.4)$ \\
\hline Healthcare personnel, n(\%) & $6(7.9)$ \\
\hline \multicolumn{2}{|l|}{ Comorbidities } \\
\hline Hypertension, $\mathbf{n}(\%)$ & $4(5.3)$ \\
\hline Diabetes, n (\%) & $6(7.9)$ \\
\hline Asthma, n (\%) & $6(7.9)$ \\
\hline \multicolumn{2}{|l|}{ Symptoms at presentation } \\
\hline Fever, n (\%) & $27(35.5)$ \\
\hline Cough, n (\%) & $27(35.5)$ \\
\hline Sore throat, n (\%) & $23(30.3)$ \\
\hline Dyspnoea, n (\%) & $7(9.2)$ \\
\hline Myalgia, n (\%) & $44(57.9)$ \\
\hline Headache, n (\%) & $41(53.9)$ \\
\hline Nausea, n (\%) & $15(19.7)$ \\
\hline Vomiting, $\mathbf{n}(\%)$ & $7(9.2)$ \\
\hline Diarrhea, $\mathbf{n}(\%)$ & $12(15.8)$ \\
\hline Fatigue, n (\%) & $50(65.8)$ \\
\hline Hiposmia, n (\%) & $24(31.6)$ \\
\hline Loss of taste, $n(\%)$ & $26(34.2)$ \\
\hline
\end{tabular}

(Abbreviations: $S D$ standard deviation)

of $\mathrm{N}$ gene $\mathrm{Ct}$ value of NSS taken by healthcare worker is $25.3 \pm 4.5$ and the mean of the RdRP gene is $25.9 \pm 5.1$. The mean $\mathrm{N}$ Gene $\mathrm{Ct}$ value of the NSS taken by the patients was $24.4 \pm 6.0$ and the mean RdRP Gene was $24.1 \pm 6.0$. The mean of $\mathrm{N}$ Gene $\mathrm{Ct}$ value of Saliva samples is $27.9 \pm 6.3$ and the average of RdRP Gene is $27.3 \pm 6.4$. The sensitivity of the three methods in the diagnosis of the COVID-19 was $100 \%, 98.7 \%$, and $96.1 \%$, respectively. The $\mathrm{Ct}$ values of the $\mathrm{N}$ and RdRP genes of these 3 different sample types were categorically examined (38 cut-off values). $\mathrm{N}$ gene sensitivity of NSS taken by healthcare worker was $100 \%$, while RdRP gene was $98.7 \%$; $\mathrm{N}$ gene sensitivity of the samples taken by the patient was $97.4 \%$, while the RdRP gene was $98.7 \%$. In addition, the sensitivity of the $\mathrm{N}$ gene of saliva samples taken by the patient was $90.8 \%$, while the sensitivity

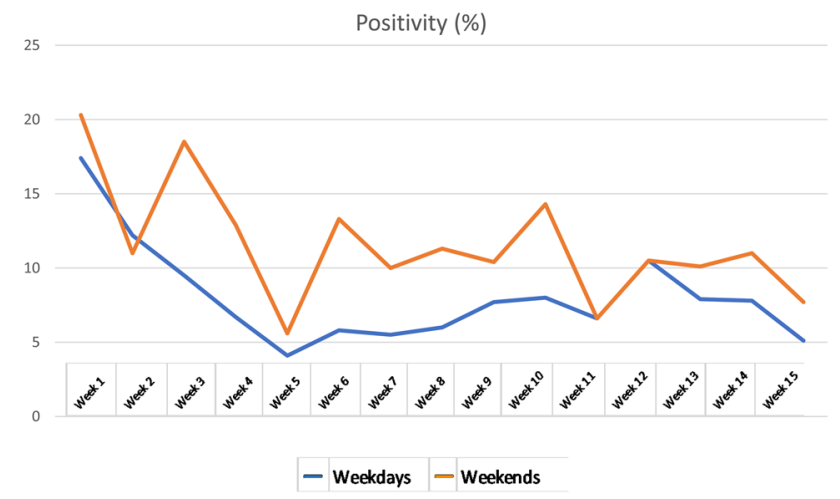

Fig. 2 Weekdays and weekends positivity rates of COVID-19

of the RdRP gene was $93.4 \%$. All the sensitivity of the samples collected by the patients contained $90 \%$. During the study, the samples taken by the healthcare worker was compared with the results of the samples taken by the patient himself. The results of the samples were compared over two different periods for the weekend and weekdays, while samples were taken at different times of pandemic intensity (Fig. 2). In the first period, 972 (13.19\%) of 7371 samples received during the weekdays and $218(16.16 \%)$ of 1346 samples received at the weekend were positive $(p=0.003$, $95 \% \mathrm{Cl}=1.08-1.49, \mathrm{OR}=1.27)$. In the second period, 1642 $(7.35 \%)$ of 22,334 samples received during the weekdays and 419 (9.54\%) of 4391 samples received at the weekend were positive $(\mathrm{p}<0.001,95 \% \mathrm{Cl}=1.19-1.49, \mathrm{OR}=1.33)$. In the first period, the rate of positivity is significantly higher than in the second period ( $p<0.001$; Mantel-Haenszel Chi-squared test), but there was no significant difference in comparing the odds ratios of both two periods $(\mathrm{p}=0.641$; Woolf's test) (Table 4).

\section{Discussion}

SARS-CoV-2 has become a global health threat. As of 29 March 2021, there have been 239,437,517 confirmed cases of COVID-19, including 4,879,235 deaths [9]. There is a constant need for HCWs in patient-facing roles in the coming global epidemic. Because this study requires close personal exposure to patients with the virus, frontline HCWs
Table 3 Healthcare worker, patient self-collected nasopharyngeal swab samples and saliva samples COVID-19 PCR reaction results

\begin{tabular}{|c|c|c|c|c|c|c|}
\hline & \multirow{2}{*}{\multicolumn{2}{|c|}{$\frac{\text { Healthcare worker }}{\text { Nasopharyngeal swab }}$}} & \multicolumn{4}{|c|}{ Patient self-collected } \\
\hline & & & \multicolumn{2}{|c|}{ Nasopharyngeal swab } & \multicolumn{2}{|l|}{ Saliva } \\
\hline & $\mathrm{N}$ gene & RdRP gene & $\mathrm{N}$ gene & RdRP gene & $\mathrm{N}$ gene & RdRP gene \\
\hline Positive & 76 & 75 & 74 & 75 & 69 & 71 \\
\hline Negative & 0 & 1 & 2 & 1 & 7 & 5 \\
\hline
\end{tabular}


Table 4 Weekdays and weekends results of two different periods in the pandemic

\begin{tabular}{|c|c|c|c|c|c|c|c|c|}
\hline & \multicolumn{3}{|c|}{ Weekdays } & \multicolumn{3}{|c|}{ Weekends } & \multirow[b]{2}{*}{$\mathrm{p}$} & \multirow[b]{2}{*}{ Difference $\%$} \\
\hline & Total (n) & Positive (n) & $\%$ & Total (n) & Positive (n) & $\%$ & & \\
\hline First period & 7371 & 972 & 13.19 & 1349 & 218 & 16.16 & 0.003 & 2.97 \\
\hline Second Period & 22,334 & 1642 & 7.35 & 4391 & 419 & 9.54 & 0.0001 & 2.19 \\
\hline
\end{tabular}

are at high risk of infection, which may contribute to further spread. The primary cause of HCWs being infected is accompanied by insufficient data on SARS-CoV-2, including virulence factors, non-host survival, resistant strains, incubation time, and infection pathophysiology. Therefore, it causes transmission of HCWs and individuals from healthcare workers. To protect against COVID 19 infections, it is necessary to use special PPE such as respirators, N-95 masks, non-perforated gowns, and visors or face shields. It has not been easy to obtain these necessary PPE against global infections. Also, PPE is often disposable and should be disposed of with the highest precautions to prevent contamination. Therefore, the inadequate availability and improper use of PPE is a critical factor contributing to the increased risk of HCWs for COVID 19 infection [10, 11]. In addition to all this, the stressful work environment, long working hours leading to fatigue, and psychological problems associated with isolation also contribute to the increased likelihood of HCWs infection of COVID-19 [12].

Other factors that may predispose HCWs to infection may include inadequate cleaning and non-disinfection of hospital surfaces, lack of viral pandemic-related training in disinfection of medical equipment [13]. In a previous study, costeffectiveness modeling revealed that using PPE developed for all patients would be a high cost in the pandemic. On the other hand, it has shown that HCWs are physically and psychologically very difficult to work under stress, difficult conditions, and long working hours with protective equipment [14-16].

In one study, the meta-analysis found that the diagnostic sensitivity for saliva nucleic acid amplification testing is approximately $83.2 \%$ (95\% CI, 74.7-91.4\%), which is comparable to that reported for nasopharyngeal swab nucleic acid amplification testing and to the result obtained using our latent class model analysis (84.8\%; 95\% CI, 76.8-92.4\%). In addition, given the ease of sampling and increased patient comfort, an important positive aspect of the reduced burden on test centers should be considered [17]. Wyllie et al. detected that nasopharyngeal sampling may be an explanation for false negative results, so monitoring an internal control for proper sample collection may provide an alternative evaluation technique. In specimens collected from inpatients by HCWs, greater variation in human RNase P Ct values in nasopharyngeal swab specimens (standard deviation, $2.89 \mathrm{Ct}$; $95 \% \mathrm{CI}, 26.53$ to 27.69 ) than in saliva specimens (standard deviation, $2.49 \mathrm{Ct}$; $95 \% \mathrm{CI}, 23.35$ to 24.35 ) has been found. When HCWs collected their own specimens, also they found greater variation in RNase P Ct values in nasopharyngeal swab specimens (standard deviation, 2.26 $\mathrm{Ct}$; $95 \% \mathrm{CI}, 28.39$ to 28.56 ) than in saliva specimens (standard deviation, $1.65 \mathrm{Ct}$; $95 \% \mathrm{CI}, 24.14$ to 24.26). Collection of saliva samples by patients themselves negates the need for direct interaction between HCWs and patients. They showed that interaction is a source of major testing bottlenecks and presents a risk of nosocomial infection. Collection of saliva samples by patients themselves also alleviates demands for supplies of swabs and personal protective equipment [18].

In this study, we detected that 76 of 132 patients were diagnosed with COVID-19 by nasopharyngeal swab (taken by the healthcare worker and the patient) or by RT-PCR from saliva samples. RT-PCR results were positive in all samples taken by healthcare worker, NSS taken by the patient in 75 patients, and saliva samples in 73 patients. Analyses were performed by comparing each sample taken by the healthcare worker with the sample taken by the patient. Three different samples from 132 patients (nasopharyngeal taken by the healthcare worker, nasopharyngeal and saliva taken by the patient), the sensitivity of the three samples in the diagnosis of the COVID-19 was (100\%, 98.7\%, and $96.1 \%$, respectively) accepted to be accurate. The results were evaluated with a $95 \%$ confidence interval and the results that are of statistically significant level was defined as $\mathrm{p}<0.05$. The sensitivity of the three methods in the diagnosis of the COVID-19 was $100 \%, 98.7 \%$, and $96.1 \%$, respectively.

The results of the self-samples taken by the patients during the study were compared with the results of the samples taken by the HCWs. Compared to the period in which patients received their own nasal samples and the positivity rates taken in other periods, no discrepancy was found in the results of the nasal samples taken by the patients themselves. The sample data of nasal samples taken by the HCWs and the positive rates of samples taken by patients were found to be compatible. This method can be used as an easier sample purchase, which reduces the workload of hospital employees. Sample collection through saliva or nasopharyngeal swabbing does not differ significantly in sensitivity and less costly alternative that could replace nasopharyngeal swabs for collection of clinical samples for SARS-CoV-2 testing [19].

This study demonstrated the clinical usefulness of nasopharyngeal samples collected by HCWs and nasopharyngeal or saliva samples collected by patients for the diagnosis of COVID-19. It has been shown that samples 
taken by patients during this challenging pandemic period can provide more comfortable patient experience. Besides, droplets from frequent coughing or sneezing during sampling increase the risk of other people becoming infected. Contact between COVID-19 patients causes respiratory infections in HCWs [20]. It also reduces the use of personnel protective equipment and especially the need for personnel sampling is important. Also, it is easy to screen for COVID-19 in large areas such as airports or home and office-based testing of asymptomatic patients. Our study is very important due to the increasing intensity of the COVID-19 epidemic during this period, and it is not known exactly when it will end. We think self-collected nasopharyngeal and saliva samples are a useful approach during the COVID-19 outbreak. Ultimately, in a rapidly changing pandemic, it is essential that sampling strategies are adapted to real-life experience.

Funding This study was carried out with the contribution of the İstanbul University-Cerrahpaşa Scientific Research and Project Fund (Grant No: TSA-2020-34955).

\section{Declarations}

Ethics approval and consent to participate Ethical approval was taken from Non-Pharmaceutical Clinical Research Ethics Committee of İstanbul University-Cerrahpaşa, Cerrahpaşa Faculty of Medicine (Date: 21.05.2020 and Decision No:83045809-604.01.02-A19). All procedures were conducted by the recommendations of the Declaration of Helsinki. All authors contributed to the design, data collection, analysis, and final version of the study.

Informed consent Is signed by all patients prior to inclusion.

Conflict of interest The authors declare no competing interests.

\section{References}

1. WHO COVID-19 Explorer. Geneva: World Health Organization (2021) https://worldhealthorg.shinyapps.io/covid/. Accessed 17 October 2021

2. Gómez-Ochoa SA, Franco OH, Rojas LZ et al (2021) COVID-19 in health-care workers: a living systematic review and meta-analysis of prevalence, risk factors, clinical characteristics, and outcomes. Am J Epidemiol 190(1):161-175. https://doi.org/10.1093/aje/kwaa191

3. Tu YP, Jennings R, Hart B et al (2020) COVID-19: protecting health-care workers. Lancet 395(10228):922. https://doi.org/10. 1016/S0140-6736(20)30644-9

4. Karlsson U, Fraenkel CJ (2020) Covid-19: risks to healthcare workers and their families. BMJ 371:m3944. https://doi.org/10.1136/bmj. m3944

5. Malhotra N, Gupta N, ISH S, Ish P (2020) COVID-19 in intensive care. Some necessary steps for health care workers. Monaldi Arch Chest Dis 25:90(1). https://doi.org/10.4081/monaldi.2020.1284
6. Qian Y, Zeng T, Wang $\mathrm{H}$ et al (2019) Safety management of nasopharyngeal specimen collection from suspected cases of coronavirus disease 2019. J Nurs Sci 7(2):153-156. https://doi.org/10.1016/j. ijnss.2020.03.012

7. World Health Organization Interim guidance (2021) Infection prevention and control during health care when coronavirus disease (COVID-19) is suspected or confirmed. https://www.who.int/publications/i/item/ WHO-2019-nCoV-IPC-2021.1 (accessed 12 July 2021)

8. Tu YP, Jennings R, Hart B et al (2020) N. Swabs collected by patients or health care workers for SARS-CoV-2 testing. N Engl J Med 383(5):494-496. https://doi.org/10.1056/NEJMc2016321

9. https://covid19.who.int/table. Live Dashboard 13 Oct 2021

10. Xiang YT, Jin Y, Wang Y (2020) Tribute to health workers in China: a group of respectable population during the outbreak of the COVID-19. Int J Biol Sci 16(10):1739-1740. https://doi.org/10. $7150 /$ ijbs. 45135

11. Bauchner H, Fontanarosa PB, Livingston EH (2020) Conserving supply of personal protective equipment - a call for ideas. JAMA 323(19):1911. https://doi.org/10.1001/jama.2020.4770

12. Wang J, Zhou M, Liu F (2020) Reasons for healthcare workers becoming infected with novel coronavirus disease 2019 (COVID19) in China. J Hosp Infect 105(1):100-101. https://doi.org/10. 1016/j.jhin.2020.03.002

13. Otter JA, Yezli S, French GL (2011) The role played by contaminated surfaces in the transmission of nosocomial pathogens. Infect Control Hosp Epidemiol 32(7):687-99. https://doi.org/10.1086/ 660363

14. Mukerji S, MacIntyre CR, Seale H (2017) Cost-effectiveness analysis of $\mathrm{N} 95$ respirators and medical masks to protect healthcare workers in China from respiratory infections. BMC Infect Dis 17(1):464. https://doi.org/10.1186/s12879-017-2564-9

15. Thomas JP, Srinivasan A, Wickramarachchi CS et al (2020) Evaluating the national PPE guidance for NHS healthcare workers during the COVID-19 pandemic. Clin Med 242-7. https://doi.org/10.7861/ clinmed.2020-0143

16. Wei X, Lin F, Haihan L et al (2020) The physical and psychological effects of personal protective equipment on health care workers in Wuhan, China: a cross-sectional survey study. J Emerg Nurs 791-801.e7. https://doi.org/10.1016/j.jen.2020.08.004

17. Butler-Laporte G, Lawandi A, Schiller I et al (2021) Comparison of saliva and nasopharyngeal swab nucleic acid amplification testing for detection of SARS CoV-2 a systematic review and metaanalysis. JAMA Intern Med 181(3):353-360. https://doi.org/10. 1001/jamainternmed.2020.8876.

18. Wyllie AL, Fournier J, Casanovas-Massana A et al (2020) Saliva or nasopharyngeal swab specimens for detection of SARS-CoV-2. N Engl J Med 383(13):1283-1286. https://doi.org/10.1056/NEJMc2016359

19. Bastos ML, Perlman-Arrow S, Menzies D et al (2021) The sensitivity and costs of testing for SARS-CoV-2 infection with saliva versus nasopharyngeal swabs: a systematic review and meta-analysis. Ann Intern Med 12:M20-6569. https://doi.org/10.7326/L21-0055

20. World Health Organization (2014) Infection prevention and control of epidemic- and pandemic-prone acute respiratory infections in health care. https://www.who.int/publications-detail/infectionprevention-and-control-during-health-care-when-novel-coronavirus(ncov)-infection-is-suspected-20200125 (accessed 30 March 2021)

Publisher's Note Springer Nature remains neutral with regard to jurisdictional claims in published maps and institutional affiliations. 